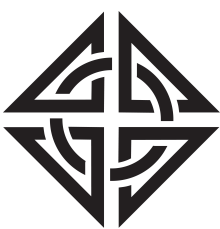

SCIENTIA
Sharif University of Technology

Scientia Iranica

Transactions D: Computer Science \& Engineering and Electrical Engineering http://scientiairanica.sharif.edu

\title{
Multi-verse optimization algorithm for optimal synthesis of phase-only reconfigurable linear array of mutually coupled parallel half-wavelength dipole antennas placed at finite distances from the ground plane
}

\author{
D. Jamunaa ${ }^{a, *}$, G.K. Mahanti ${ }^{a}$, and F.N. Hasoon ${ }^{b}$ \\ a. Department of Electronics and Communication Engineering, National Institute of Technology, Durgapur-713209, India. \\ b. Department of Electrical and Communication Engineering, College of Engineering, NUST, Sultanate of Oman.
}

Received 30 April 2019; received in revised form 16 April 2020; accepted 26 October 2020

KEYWORDS
Reconfigurable linear
array antenna;
Multi-verse
optimization
algorithm;
Mutual coupling;
Voltage standing wave
ratio;
Particle swarm
optimization;
Grey wolf
optimization;
Imperialist
competitive
optimization
algorithms.

\begin{abstract}
In the case of antenna arrays, researchers usually neglect the effect of mutual coupling of antennas placed in proximity to each other. The interchange of electromagnetic energy between an antenna and a far-field point depends on not only the transmitting antenna, but also its neighboring antennas. This effect is referred to as mutual coupling between dipole antenna elements and is considered here in the synthesis of phase-only reconfigurable antenna arrays. The main objective of this work is to produce the desired side lobe level and voltage standing wave ratio, in addition to few other radiation pattern parameters. Multi-verse Optimization algorithm is employed for the purpose of generating voltage amplitude and discrete phase distributions in the dipole elements to generate flattop beam/pencil beam patterns. These two patterns share common amplitude distributions and differ in phase distributions. Results of simulations proved that this algorithm accomplished its task successfully and was superior to other algorithms like particle swarm optimization, grey wolf optimization, and imperialist competitive optimization algorithms.
\end{abstract}

(C) 2022 Sharif University of Technology. All rights reserved.

\section{Introduction}

Recent developments in the field of communications demand a more flexible and convenient approach to the provision of multi-beam patterns. In this regard,

\footnotetext{
*. Corresponding author.

E-mail address: jamunaad@yahoo.com (D. Jamunaa); gautammahanti@yahoo.com (G.K. Mahanti); ferasalattar@nu.edu.om (F.N.Hasoon)
}

antenna arrays $[1,2]$ guarantee obtaining such patterns by providing common and different excitations. These arrays are referred to as reconfigurable arrays [1-9] and the corresponding excitations include amplitudes, phases, positions, etc. Literature review reports the viability of many methods including projection approach [3], Woodward Lawson synthesis [4], and other evolutionary algorithms [5-8] to generate the beams.

A number of concerns hinder the effective generation of these beams. One of these concerns is mutual coupling [10-13], which plays a prominent role in diminishing the radiation pattern of any antenna array. 
This mutual coupling refers to electromagnetic interactions within the neighboring elements existing in an antenna array. In simple words, it is said that the electric field generated by an antenna element affects the neighboring elements such that the total pattern gets deviated from the desired one. In addition, relative separation between the elements as well as the orientation of the elements influence the mutual coupling effects.

To add to the concern above, an effective mismatch between an antenna and the feeding network hinders perfect transreception. A mismatch between the antenna and the feeder line or the subsequent components results in return loss. Necessary care is taken in this paper to include both the mutual coupling effects as well as sustained standing wave ratio. Simulations are conducted following the inclusion of ground plane and their analysis targets the effect of the distance between the ground plane and the antenna array.

To implement this process, evolutionary algorithms are used here to generate voltage amplitudes that can hold values between 0 and 1 and current amplitudes are calculated using the mutual impedance matrix. These algorithms also generate phase excitations that may vary between $-180^{\circ}$ and $180^{\circ}$ in discrete steps using a 6-bit phase shifter [5].

Multi-verse optimization algorithm [14-22] was applied to the generation of required amplitudes as well as phase excitations. The reason for choosing multi-verse optimization algorithm lies in its immense success in providing solutions to problems related to antenna arrays, especially in the synthesis of large arrays. The performance of this algorithm is investigated numerically and compared with few other standard popular algorithms, namely Particle Swarm Optimization (PSO) [23,24], Imperialist Competitive Algorithm (ICA) [25-29], and Grey Wolf Optimization (GWO) [30-33]. All the algorithms used in this paper are run to minimize the fitness value in the weighted fitness functions to achieve the desired pattern. The novelty of this paper is that voltage standing wave ratio is considered simultaneously for both flat-top and pencil beams. Mutual coupling effect is taken into account along with the ground plane effects. Also, the phase excitations are controlled by discrete phase shifters which greatly reduce the complexity of feed networks.

\section{Theory}

The free space far-field pattern of a linear array constructed of $N$ half-wave dipoles is separated from each other by a distance $d$ on the azimuth plane with $\emptyset$ being the azimuth angle measured from the $x$-axis, as shown in Figure 1. This is given by the following relation.

$$
F(\emptyset)=\sum_{n=1}^{N} I_{n} e^{j(n-1) k d \cos \emptyset} * E P(\emptyset),
$$

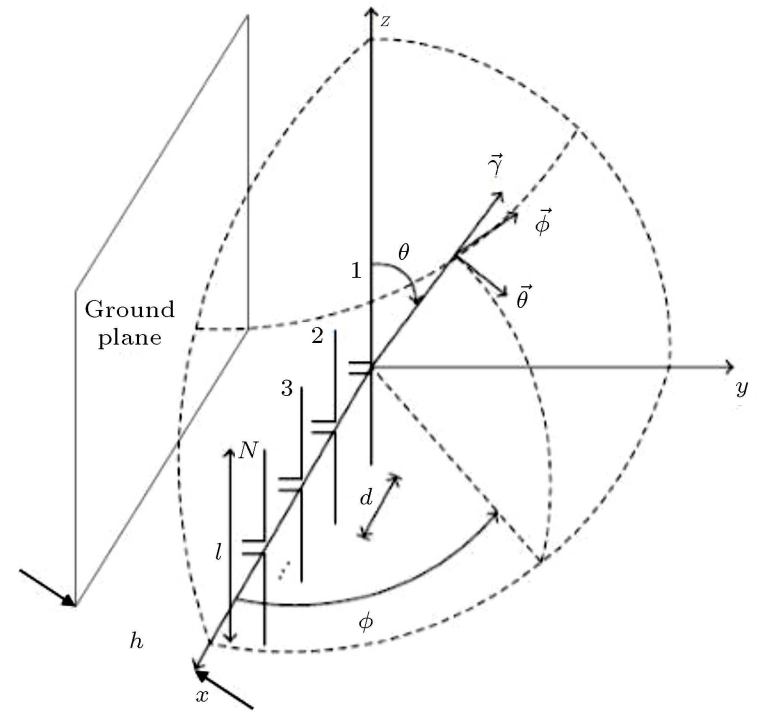

Figure 1. A linear array of parallel half-wavelength dipole antennas with ground plane placed at a distance $h$ behind the array.

where $k$ is the wave number, $\operatorname{EP}(\emptyset)$ the element pattern, and $I_{n}$ the complex current excitation obtained from the combination of impedance matrix and the voltage excitation matrix of the elements.

$$
[I]_{N * 1}=[Z]_{N * N}^{-1}[V]_{N * 1},
$$

where $[Z]$ is the impedance matrix (size $N^{*} N$ ) and $[V]$ is the voltage excitation matrix (size $N^{*} 1$ ) of the elements.

The element pattern of the dipole elements is assumed to be omnidirectional in the plane considered, i.e., $\operatorname{EP}(\emptyset)=1$. From the currents calculated using the voltage excitations as well as the impedance matrix, a sum pattern is generated in the broadside direction.

Since mutual coupling effects are included in this paper, the customary equations related to it are shown as follows. The mutual coupling includes both the self-impedance of the elements as well as the mutual impedances among elements [2]. The relationship between the voltages $V$ and impedances is given by:

$$
V_{p}=I_{p} Z_{p p}+\sum_{p \neq q} I_{q} Z_{p q}
$$

where $Z_{p p}$ refers to self-impedance of dipole $p$ and $Z_{p q}$ is the mutual impedance between $p$ and $q$. The active impedance is given by:

$$
Z_{p}^{A c}=Z_{p p}+\sum_{p \neq q}\left(I_{q} / I_{p}\right) Z_{p q}
$$

In case a ground plane [2] is kept at distance $h$ behind the array, the new active impedance is calcu- 
lated considering the image principles in obtaining the impedances of the elements.

In the impedance matrix, self-impedance and mutual impedance are replaced by $\left(Z_{p p}-Z_{p p}^{*}\right)$ and $\left(Z_{p q}-Z_{p q}^{*}\right)$, respectively, where $Z_{p p}^{*}$ is the impedance between the $p$ th dipole and its image and $Z_{p q}^{*}$ is the impedance between the $p$ th dipole and image of the $q$ th dipole. If $h$ is the distance between the array and the ground plane and if the element factor is $\sin (k h \sin \emptyset)$, then a new far-field expression taking the ground plane into effect is given by:

$$
F(\emptyset)=\sum_{n=1}^{N}[\sin (k h \sin \emptyset)] I_{n} e^{j(n-1) k d \cos \emptyset},
$$

$$
\text { Normalized absolute far-field }=\frac{|F(\emptyset)|}{\max |F(\emptyset)|} .
$$

Voltage Standing Wave Ratio (VSWR) is calculated as $(1+\Gamma) /(1-\Gamma)$, where $\Gamma$ is the reflection coefficient equal to $\left(Z_{p}^{A c}-Z_{0}\right) /\left(Z_{p}^{A c}+Z_{0}\right)$. $Z_{0}$ is the characteristic impedance with a value of $50 \Omega$. The small value of VSWR denotes a good matching condition of the array. The weighted fitness function is given by:

$$
F=\sum_{i=1}^{3} w_{i}\left(F_{i}\right)^{2}+\sum_{j=1}^{2} w_{j}\left(F_{j}\right)^{2}
$$

The first term in the Right Hand Side (RHS) of the above equation is written below:

$$
F_{i}=\left\{\begin{array}{lll}
F_{i}^{\text {flat }}-F_{i, d}^{\text {flat }}, & \text { if } & F_{i}^{\text {flat }}>F_{i, d}^{\text {flat }} \\
0, & \text { if } \quad F_{i}^{\text {flat }} \leq F_{i, d}^{\text {flat }}
\end{array}\right.
$$

where $F_{i}$ represents the flat-top beam parameter and $i=1,2$ and 3 represent such parameters as Side Lobe Level (SLL) in $\mathrm{dB}$, ripple in $\mathrm{dB}$, and maximum VSWR (no unit).

The second term in the RHS of Eq. (7) is written as follows:

$$
F_{j}= \begin{cases}F_{j}^{p e n}-F_{j, d}^{p e n}, & \text { if } F_{j}^{p e n}>F_{j, d}^{p e n} \\ 0, & \text { if } F_{j}^{p e n} \leq F_{j, d}^{p e n}\end{cases}
$$

where $F_{j}$ represents the pencil beam parameter and $j=1$ and 2 include such parameters as SLL in dB and maximum VSWR (no unit).

Moreover, in the above equations, the superscript pen represents the specifications of the pencil pattern and the superscript flat represents the specifications of the flat-top pattern. The terms $F_{i d}$ and $F_{j d}$ represent the expected/desired values, and $F_{i}$, and $F_{j}$ represent the obtained values for each specification parameter. The weights $w$ are equal to 1 , suggesting that equal importance is given to all the parameters during the optimization process.

\section{Multi-verse optimization algorithm}

Multi-Verse Optimization (MVO) algorithm is one of the recently introduced algorithms that is influenced by the multi-verse theory concepts. As per the concepts dealing with this algorithm, our universe may be one of the infinite number of universes that may exist. The theory underlying this fact is dependent on white holes, black holes, and wormholes. In this algorithm, the worm holes are responsible for exploitation and the combined white and black holes control the exploration part. Here, a solution represents a universe; a variable refers to an object in it; inflation rate is the fitness value of the solution; and the term time refers to the iteration. The rules used in this algorithm are given below:

(i) The higher inflation rate indicates the situation having more white holes than black holes;

(ii) The universes with a higher inflation rate move the objects through the white holes and the universes with a lower inflation rate accept the objects via the black holes;

(iii) The objects in all universes move randomly towards the best universe via wormholes regardless of the effect of the inflation rate.

The objects travel between universes through the white or black hole tunnels. In the creation of a tunnel between two universes, the universe with a higher inflation rate is treated as a white hole, while another universe is a black hole. Then, the objects are allowed to move from the white holes of one universe to the black holes of another. Thus, exchange of objects can easily take place without any hassle. Moreover, as assumed, when the inflation rate is higher, the probability of having white holes is greater.

Wormholes appear in a random manner in any of the universes irrespective of the inflation rate. This ensures a greater diversity of universes during iterations. The tunnels require universes to change in an abrupt manner, thus guaranteeing exploration of the search within the allotted space. These changes facilitate relieving any local optimum stagnation. The wormholes also indulge in re-spanning of few variables around the best obtained solution in a random way, thus ensuring exploitation around the most promising region.

Mathematical modeling of the interchange of the objects between the universes and the white and black hole tunnels is done through roulette wheel selection. At the end of every iteration, one universe is chosen as the best one. Given that $d$ and no represent the number of variables and universes, respectively, the set of solutions $U$ is formulated as follows: 
$x_{m}^{n}=\left\{\begin{array}{lll}\left\{X_{n}+\operatorname{TDR}\left(\left(U r_{n}-L r_{n}\right) \operatorname{ran} 4+L r_{n}\right)\right. & \operatorname{ran3}<0.5 & \\ X_{n}-\operatorname{TDR}\left(\left(U r_{n}-L r_{n}\right) \operatorname{ran} 4+L r_{n}\right) & \operatorname{ran} 3 \geq 0.5 & \\ x_{m}^{n} & \text { ran2 } \geq W E P\end{array}\right.$

Box I

$$
U=\left[\begin{array}{ccccc}
x_{1}^{1} & x_{1}^{2} & x_{1}^{3} & \cdots & x_{1}^{d} \\
\vdots & \vdots & \vdots & \cdots & \vdots \\
\vdots & \vdots & \vdots & \cdots & \vdots \\
\vdots & \vdots & \vdots & \cdots & \vdots \\
x_{n o}^{1} & x_{n o}^{2} & x_{n o}^{3} & \cdots & x_{n o}^{d}
\end{array}\right]
$$

If $U m$ is the $m$ th universe, $N I r(U m)$ is the normalized inflation rate of the $m$ th universe; then, we have:

$$
x_{m}^{n}= \begin{cases}x_{k}^{n} & \operatorname{ran} 1<N \operatorname{Nr}\left(U_{m}\right) \\ x_{m}^{n} & \operatorname{ran} 1 \geqslant N \operatorname{Nr}\left(U_{m}\right)\end{cases}
$$

where $\operatorname{ran} 1$ is a random number between 0 and 1 and $x_{k}^{n}$ is the $n$th parameter of the $k$ th universe selected through the selection method. The facility is employed so as to make use of the changes locally for every universe. Furthermore, to upgrade the inflation rate, the wormhole tunnels are created between a Universe and the Best universe obtained up to that time and Eq. (11) shown in Box I is used for the same, where $X_{n}$ is the $n$th parameter of the Best Universe obtained so far, $T D R$ is the travelling distance rate, $W E P$ is the probability of worm hole existence, $L r_{n}$ and $U r_{n}$ are the lower and upper bounds of the $n$th variable, $x_{m}^{n}$ is the $n$th parameter of the $m$ th universe, and ran2, ran3, and ran 4 are random numbers between 0 and 1 . WEP is given as follows:

$$
W E P=m n+l\left(\frac{m x-m n}{\max \_ \text {Iters }}\right),
$$

$$
T D R=1-\frac{l^{1 / p}}{\max \_\operatorname{Iter} s^{1 / p}},
$$

where $m n$ is set to $0.2, m x$ is set to $1, l$ is the current iteration, $\max _{\text {Iters }}$ refers to the maximum iterations, and $p$ is the accuracy of the process of exploitation over iterations. The pseudo code is found as shown in Figure 2.

\section{Simulation results and discussion}

A total of 20 elements are used in this simulation process. Because of symmetry, it is made customary for the algorithms to generate only 10 element excitations. Here, the amplitudes are kept common to both of the beams, whereas the generated discrete phases are used to produce a flat-top beam and zero phases to produce a pencil beam. The amplitudes range from 0 to 1 and the phases range from $-180^{\circ}$ to $180^{\circ}$. The algorithm is run for a maximum of 200 iterations. The dipoles used here have a length of $0.5 \lambda$ and a radius of $0.005 \lambda$. The distance between the dipoles is kept at $0.5 \lambda$. The ground plane is taken into consideration for various distances of $0.10 \lambda, 0.20 \lambda$, and $0.25 \lambda$ for simulation purposes. The population size and maximum number of iterations are kept the same for all the algorithms. A total of five runs are used for the algorithms and the best out of the five runs based on the lowest fitness values are chosen as the final generated values of excitations. Tables 1,2 , and 3 show the parameter values for the linear array at distances of $0.10 \lambda, 0.20 \lambda$, and $0.25 \lambda$ from the ground plane.

Table 1. Parameter values for the linear array at a distance of $0.10 \lambda$ from the ground plane.

\begin{tabular}{llccccc}
\hline \multirow{2}{*}{ Patterns } & \multirow{2}{*}{ Parameters } & Desired & \multicolumn{4}{c}{ Obtained values } \\
\cline { 4 - 7 } & & values & MVO & GWO & PSO & ICA \\
\hline \multirow{2}{*}{ Pencil beam } & SLL in dB & -22 & -22.0934 & -22.0222 & -21.8158 & -22.1674 \\
& VSWR & 1.3 & 1.2359 & 1.2621 & 1.2233 & 1.374 \\
& & & & & & \\
\multirow{2}{*}{ Flat-top beam } & SLL in dB & -22 & -23.1407 & -22.1199 & -21.9791 & -21.7364 \\
& VSWR & 1.3 & 1.2444 & 1.526 & 1.8901 & 1.4504 \\
& Ripple in dB & & & & & \\
& $\left(75^{\circ}<\phi<105^{\circ}\right)$ & 0.5 & 0.57652 & 0.73797 & 1.7995 & 1.3535 \\
\hline
\end{tabular}




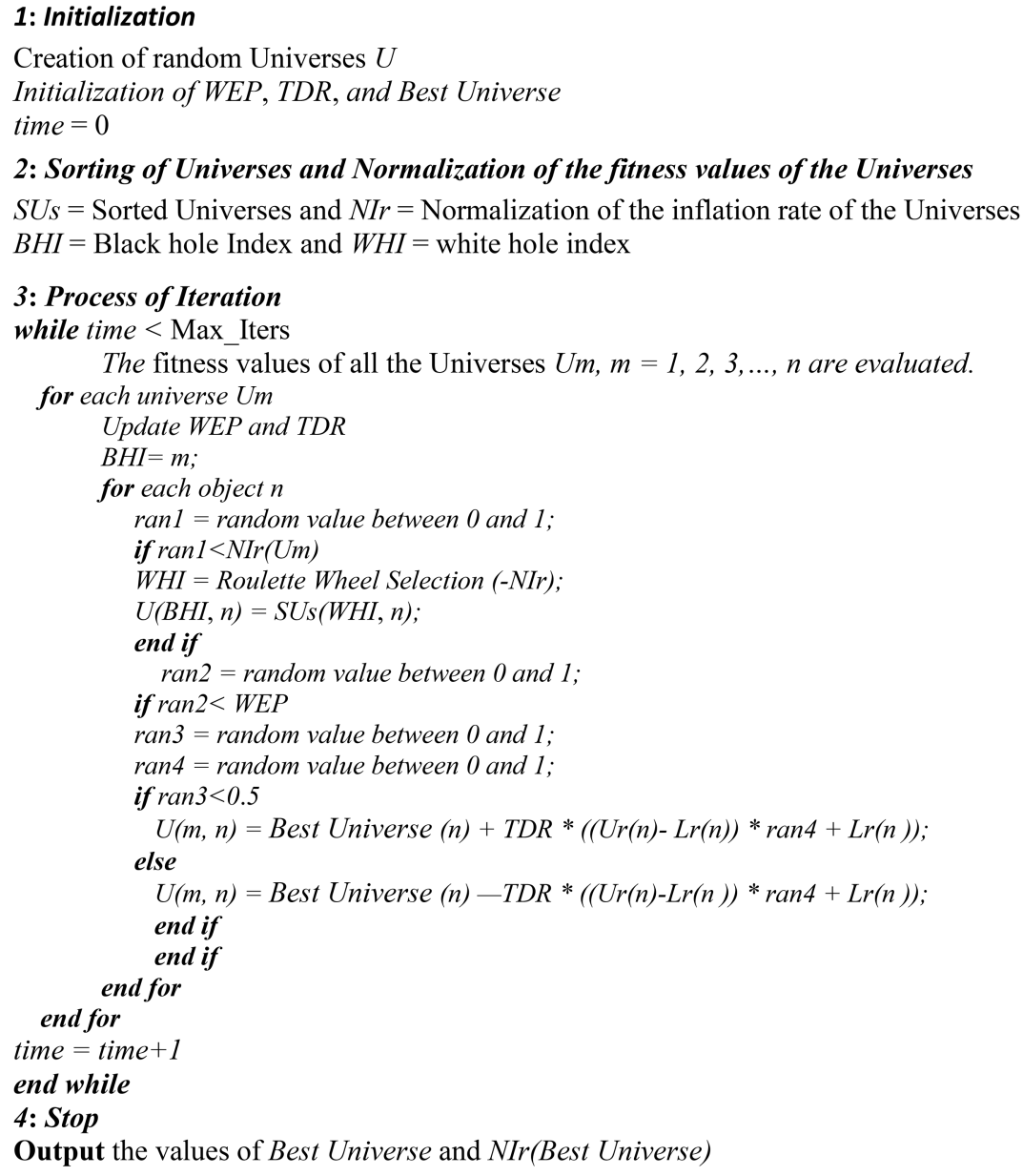

Figure 2. Pseudo code for multi-verse optimization algorithm.

Table 2. Parameter values for the linear array at a distance of $0.20 \lambda$ from the ground plane.

\begin{tabular}{llccccc}
\hline \multirow{2}{*}{ Patterns } & \multirow{2}{*}{ Parameters } & Desired & \multicolumn{4}{c}{ Obtained values } \\
\cline { 3 - 6 } vencil beam & & -22 & -22.029 & -22.0996 & -21.8788 & -22.1899 \\
& SLL in dB & 1.3 & 1.2221 & 1.3269 & 2.0011 & 1.9924 \\
& VSWR & & & & & \\
& & -22 & -23.3714 & -22.468 & -21.9851 & -22.4286 \\
\multirow{2}{*}{ Flat-top beam } & SLL in dB & 1.3 & 2.0504 & 1.2497 & 2.1046 & 2.1249 \\
& VSWR & & & & & \\
& Ripple in dB & 0.5 & 0.7899 & 0.5378 & 0.97112 & 1.3829 \\
& $\left(75^{\circ}<\phi<105^{\circ}\right)$ & & & & & \\
\hline
\end{tabular}

Table 3. Parameter values for the linear array at a distance of $0.25 \lambda$ from the ground plane.

\begin{tabular}{|c|c|c|c|c|c|c|}
\hline \multirow{2}{*}{ Patterns } & \multirow{2}{*}{ Parameters } & \multirow{2}{*}{$\begin{array}{l}\text { Desired } \\
\text { values }\end{array}$} & \multicolumn{4}{|c|}{ Obtained values } \\
\hline & & & MVO & GWO & PSO & ICA \\
\hline \multirow{2}{*}{ Pencil beam } & SLL in $\mathrm{dB}$ & -22 & -22.2765 & -22.1221 & -21.843 & -21.0699 \\
\hline & VSWR & 1.3 & 2.0704 & 2.1706 & 2.378 & 2.3361 \\
\hline \multirow{3}{*}{ Flat-top beam } & SLL in $\mathrm{dB}$ & -22 & -23.6803 & -24.4325 & -21.5715 & -20.8859 \\
\hline & VSWR & 1.3 & 2.1817 & 2.3451 & 3.1563 & 2.3944 \\
\hline & $\begin{array}{l}\text { Ripple in dB } \\
\left(75^{\circ}<\phi<105^{\circ}\right)\end{array}$ & 0.5 & 0.7977 & 0.5419 & 2.376 & 2.5608 \\
\hline
\end{tabular}




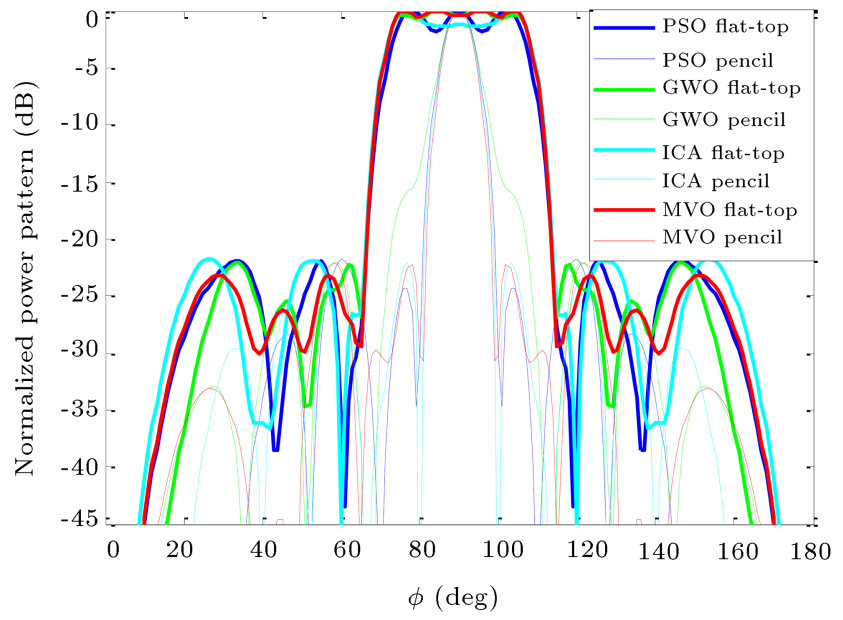

Figure 3. Normalized power in $\mathrm{dB}$ vs $\phi$ in degrees for the linear array placed at a distance of $0.10 \lambda$ from the ground plane.

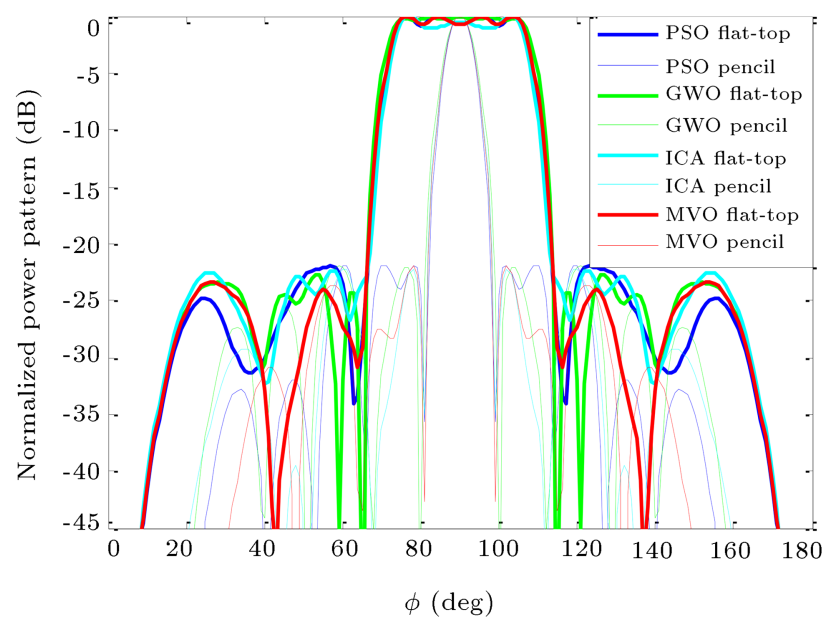

Figure 4. Normalized power in $\mathrm{dB}$ vs $\phi$ in degrees for the linear array placed at a distance of $0.20 \lambda$ from the ground plane.

Figures 3, 4, and 5 show the normalized power pattern in $\mathrm{dB}$ versus $\phi$ in degrees for the linear array with distances of $0.10 \lambda, 0.20 \lambda$, and $0.25 \lambda$ from the ground plane. Table 4 shows the corresponding voltage and phase distributions. Figure 6 shows the VSWR values for all the algorithms at different distances between the array and the plane. Table 5 shows the fitness values and computation times. Figure 7 shows the fitness values versus iteration numbers.

From Table 1, it is seen that the MVO exhibits its supremacy in delivering the best excitation values to produce the parameter values well under the expected criteria. The values of these parameters include SLL and VSWR in pencil beam and SLL and VSWR in the flat-top beam. A deficit of $0.07652 \mathrm{~dB}$ exists in the ripple in the flat-top beam. GWO managed to produce all the parameter values to the expected level except VSWR and ripple in the flat-top beam. Overall,

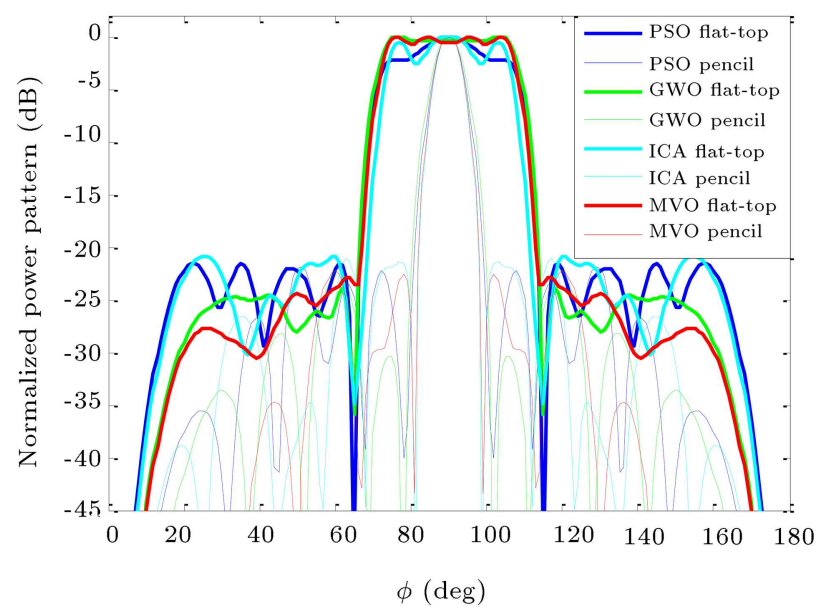

Figure 5. Normalized power in $\mathrm{dB}$ vs $\phi$ in degrees for the linear array placed at a distance of $0.25 \lambda$ from the ground plane.

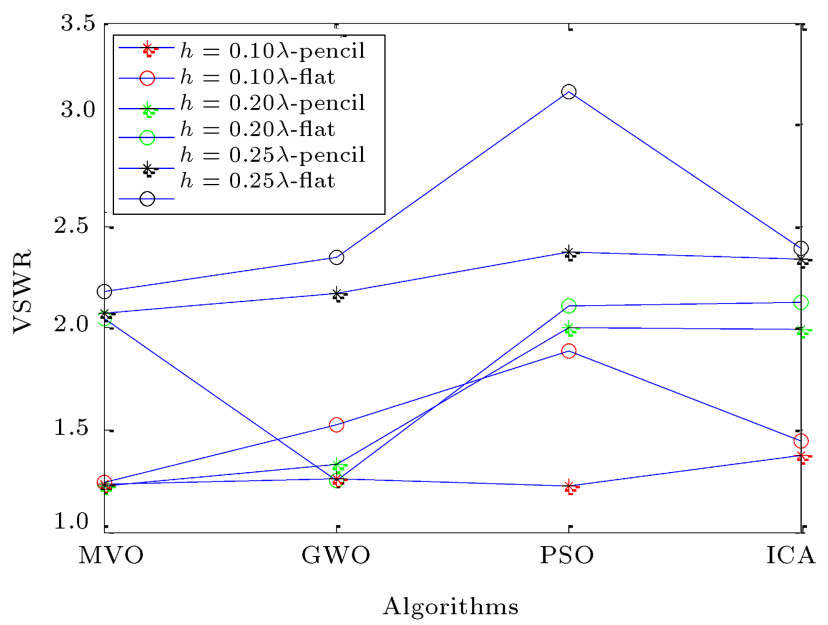

Figure 6. VSWR values versus algorithms.

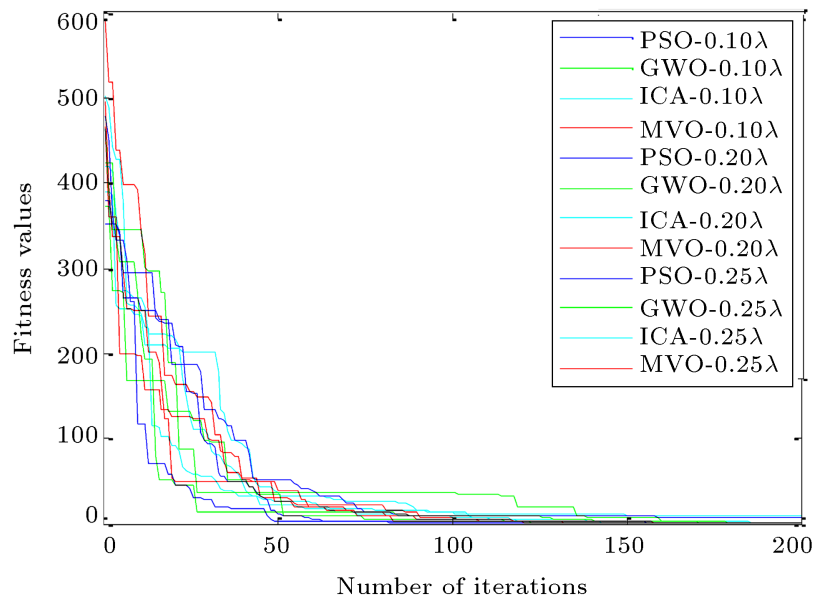

Figure 7. Fitness values versus number of iterations.

PSO and ICA are not as favorable as the remaining algorithms in terms of their outcome. Except PSO, the expected SLL value is determined by all algorithms 
Table 4. Voltage amplitude and phase distributions (in degrees) of the elements for different distances from the ground plane.

\begin{tabular}{|c|c|c|c|c|c|c|}
\hline \multirow{2}{*}{$\begin{array}{l}\text { Element } \\
\text { number }\end{array}$} & \multicolumn{2}{|c|}{$\begin{array}{c}h=0.10 \lambda \\
\text { distribution }\end{array}$} & \multicolumn{2}{|c|}{$\begin{array}{c}h=0.20 \lambda \\
\text { distribution }\end{array}$} & \multicolumn{2}{|c|}{$\begin{array}{c}h=0.25 \lambda \\
\text { distribution }\end{array}$} \\
\hline & Voltage & $\begin{array}{c}\text { Phase } \\
\text { (degrees) }\end{array}$ & Amplitude & $\begin{array}{c}\text { Phase } \\
\text { (degrees) }\end{array}$ & Amplitude & $\begin{array}{c}\text { Phase } \\
\text { (degrees) }\end{array}$ \\
\hline $1 \& 20$ & 0.0007 & -50.6250 & 0.0580 & -140.625 & 0.0692 & -180.000 \\
\hline $2 \& 19$ & 0.1220 & -28.1250 & 0.1322 & 180.0000 & 0.1740 & 174.3750 \\
\hline $3 \& 18$ & 0.2498 & -45.0000 & 0.2843 & -174.375 & 0.3317 & -174.375 \\
\hline $4 \& 17$ & 0.3675 & -78.7500 & 0.4096 & 135.0000 & 0.3884 & 140.6250 \\
\hline $5 \& 16$ & 0.3206 & -151.875 & 0.3860 & 67.5000 & 0.4583 & 78.7500 \\
\hline $6 \& 15$ & 0.7274 & -168.750 & 0.6969 & 50.6250 & 0.8557 & 50.6250 \\
\hline $7 \& 14$ & 0.8189 & 157.500 & 0.9622 & 22.5000 & 0.9994 & 33.7500 \\
\hline $8 \& 13$ & 0.8188 & 146.2500 & 0.7685 & 5.6250 & 0.8383 & 22.5000 \\
\hline $9 \& 12$ & 0.6822 & 95.6250 & 0.7921 & -39.3750 & 0.8501 & -33.7500 \\
\hline $10 \& 11$ & 0.8846 & 61.8750 & 0.8534 & -78.7500 & 0.9997 & -67.5000 \\
\hline
\end{tabular}

Table 5. Fitness values and computation time details.

\begin{tabular}{ccccc}
\hline \multirow{2}{*}{ Distance $\boldsymbol{h}$} & \multicolumn{4}{c}{ Fitness values } \\
\cline { 2 - 5 } & MVO & GWO & PSO & ICA \\
\hline 0.1 & 0.0058 & 0.1078 & 2.0711 & 0.8678 \\
0.2 & 1.0868 & 1.5703 & 1.3754 & 1.9395 \\
0.25 & 1.4596 & 1.8518 & 8.3029 & 8.6239 \\
\hline Distance $\boldsymbol{h}$ & Computational & time in & seconds \\
\hline 0.1 & 18001 & 18507 & 21730 & 22726 \\
0.2 & 18432 & 19421 & 19020 & 19529 \\
0.25 & 18389 & 17777 & 18380 & 19053 \\
\hline
\end{tabular}

for the pencil beam. Figure 3 shows the normalized power in $\mathrm{dB}$ versus $\phi$ in degrees for the linear array at a distance of $0.10 \lambda$ from the ground plane.

From Table 2, it is seen that the MVO algorithm succeeds in producing SLLs well within the expected values for both of the beams. The ripple in $\mathrm{dB}$ is very close to the expected value with a deficit of $0.2899 \mathrm{~dB}$. It faces a tough competition with GWO algorithm as it succeeds in producing the best VSWR in pencil beam, whereas it is lost to the same in flat-top beam.

From Table 3, it is found that MVO algorithm again succeeded in producing the best outputs, especially over GWO in VSWRs of both the beams. However, GWO slightly edged better over MVO by $0.2558 \mathrm{~dB}$ in the ripple portion in the flat-top beam.

Table 5 shows that the fitness values of MVO algorithm are quite lower than those of other algorithms for all the values of the distance between the array and the ground plane. It is also shown that the computational time taken by MVO is shorter in most places over other algorithms.
Figure 7 shows the plot between fitness values and number of iterations. According to this figure, MVO algorithm performed better in terms of convergence speed and had the lowest fitness value over other algorithms. Overall, MVO outperformed other algorithms. The algorithm's success is justified given that abrupt changes increase the exploration of the search space and resolve the problem of the stagnation of local optima. Since wormholes randomly re-span some of the variables around the best optimum solution, there is a good level of guarantee in exploitation around the most promising region. Adaptive WEP increases the probability of the existence of wormholes, and adaptive TDR increases the accuracy of the local search. All the above reasons justify the superiority of this algorithm to other algorithms.

\section{Analysis}

To study the effect of the inter-element distance of the array on the radiation pattern parameters, simulations are done at different inter-element distances using the obtained excitations from MVO algorithm. The results are shown in Table 6 at different inter-element distances.

According to Table 6 , when the distances are either more or less than $0.5 \lambda$, the values of all parameters obtained are not within the desired limit. For instance, at $d=0.6 \lambda$, it is the ripple value in $\mathrm{dB}$ that is affected; in case of $d=0.4 \lambda$, the VSWR values and the SLL in $\mathrm{dB}$ in the flat-top beam are affected.

Further, to confirm the outputs, the whole array is simulated using FEKO software. Through the use of FEKO, the array is simulated with a random choice of a distance equal to $0.10 \lambda$. Figure 8 shows the normalized power pattern in $\mathrm{dB}$ versus $\phi$ in degrees for the linear 
Table 6. Parameter values for the linear array for different inter-element distances.

\begin{tabular}{|c|c|c|c|c|c|}
\hline \multirow[b]{2}{*}{ Patterns } & \multirow[b]{2}{*}{ Parameters } & \multirow[b]{2}{*}{$\begin{array}{l}\text { Desired } \\
\text { values }\end{array}$} & \multicolumn{3}{|c|}{ Obtained values } \\
\hline & & & $\begin{array}{c}\text { MVO } \\
d=0.5 \lambda\end{array}$ & $\begin{array}{c}\text { MVO } \\
d=0.6 \lambda\end{array}$ & $\begin{array}{c}\text { MVO } \\
d=0.4 \lambda\end{array}$ \\
\hline \multirow{2}{*}{ Pencil beam } & SLL in $\mathrm{dB}$ & -22 & -22.0934 & -21.664 & -22.3405 \\
\hline & VSWR & 1.3 & 1.2359 & 1.1981 & 1.3182 \\
\hline \multirow{3}{*}{ Flat-top beam } & SLL in $\mathrm{dB}$ & -22 & -23.1407 & -21.1033 & -16.743 \\
\hline & VSWR & 1.3 & 1.2444 & 1.2067 & 1.4087 \\
\hline & $\begin{array}{l}\text { Ripple in dB } \\
\left(75^{\circ}<\phi<105^{\circ}\right)\end{array}$ & 0.5 & 0.57652 & -4.7205 & 0.59074 \\
\hline
\end{tabular}

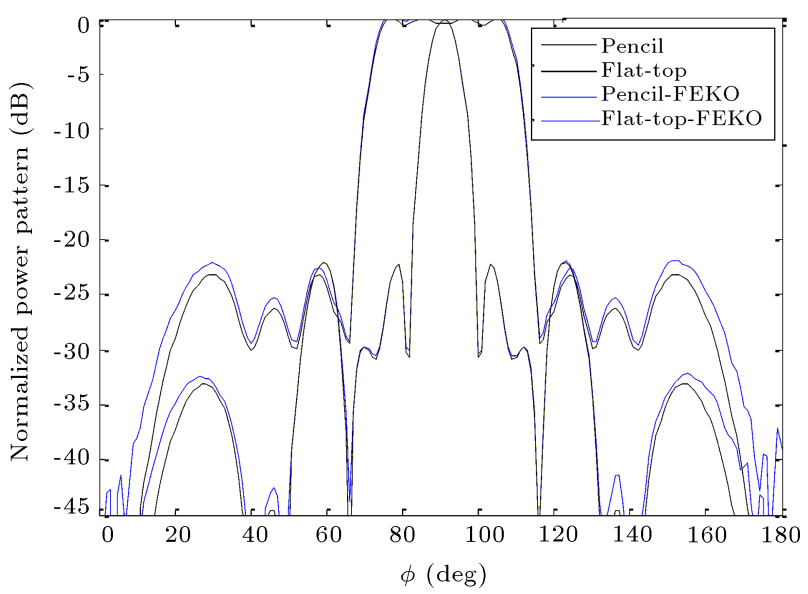

Figure 8. Normalized power in $\mathrm{dB}$ vs $\phi$ in degrees for the linear array placed at a distance of $0.10 \lambda$ from the ground plane using MATLAB simulated outputs and FEKO generated outputs.

array placed at a distance of $0.10 \lambda$ from the ground plane using MATLAB simulated outputs and FEKO generated outputs. Based on the outputs obtained, the value of SLL obtained for the flat-top pattern is found as $-21.8761 \mathrm{~dB}$ while the SLL for the pencil beam is found as $-22.0078 \mathrm{~dB}$. There is a small deficit of $0.1239 \mathrm{~dB}$ of SLL in the flat-top pattern from the desired value. Otherwise, very good agreement would be reached versus the values obtained from MATLAB outputs using MVO algorithm.

\section{Conclusion}

This study investigated the application of multi-verse optimization algorithm to reconfigurable antenna arrays. The algorithm exhibited the capability to successively generate necessary excitations in the generation of dual beams. Discrete phase shifters were used for the purpose of reducing the complexity of the feed networks. Mutual coupling was taken into account. The effect of ground plane on the radiation pattern was studied here. Moreover, real antennas were used instead of isotropic ones. Simulation results proved that MVO algorithm outperformed the compared algorithms in terms of the fitness function parameters, convergence speed, etc.

\section{References}

1. Balanis, C.A., Antenna Theory: Analysis and Design, 2nd Ed., Singapore: John Wiley and Sons (Asia) (2003).

2. Elliott, R.S., Antenna Theory and Design, New York, USA: Prentice-Hall (1981).

3. Bucci, O.M., Mazzarella, G., and Panariello, G. "Reconfigurable arrays by phase-only control", IEEE Transactions on Antennas and Propagation, 39(7), pp. 919-925 (1991).

4. Durr, M., Trastoy, A., and Ares, F. "Multiple-pattern linear antenna arrays with single prefixed amplitude distributions: modified Woodward-Lawson synthesis", Electronics Letters, 36(16), pp. 1345-1346 (2000).

5. Baskar, S., Alphones, A., and Suganthan, P.N. "Genetic algorithm based design of a reconfigurable antenna array with discrete phase shifter", Microwave and Optical Technology Letters, 45, pp. 461-465 (2005).

6. Mahanti, G.K., Chakraborty, A., and Das, S. "Design of phase-differentiated reconfigurable array antennas with minimum dynamic range ratio", IEEE Antennas and Wireless Propagation Letters, 5(1), pp. 262-264 (2006).

7. Mahanti, G.K., Das, S., Chakrabarty, A., et al. "Design of reconfigurable array antennas with minimum variation of active impedances", IEEE Antennas and Wireless Propagation Letters, 5(1), pp. 541-544 (2006).

8. Gies, D. and Rahmat-Samii, Y. "Particle swarm optimization for reconfigurable phase- differentiated array design", Microwave and Optical Technology Letters, 38, pp. 168-175 (2003).

9. Jamunaa, D., Mahanti, G.K., and Hasoon, F.N. "Synthesis of phase-only position optimized reconfigurable 
uniformly excited linear antenna arrays with a single null placement", Journal of King Saud University Engineering Sciences, 32(6), pp. 360-367 (2020).

10. Muralidharan, R., Vallavaraj, A., Mahanti, G.K., et al. "QPSO versus BSA for failure correction of linear array of mutually coupled parallel dipole antennas with fixed side lobe level and VSWR", Advances in Electrical Engineering, 2014, Article ID 858290, pp. 1-7 (2014).

11. Singh, H., Sneha, H.L., and Jha, R.M. "Mutual coupling in phased arrays", International Journal of Antennas and Propagation, 2013, Article ID 348123, pp. 1-23 (2013).

12. Lee, K.M. and Chu, R.S. "Analysis of mutual coupling between a finite phased array of dipoles and its feed network", IEEE Transactions on Antennas and Propagation, 36(12), pp. 1681-1699 (1988).

13. Muralidharan, R., Vallavaraj, A., and Mahanti, G.K. "Firefly algorithm for failure correction of linear array of dipole antennas in presence of ground plane with mutual coupling effects", ACES Journal, 30(10), pp. 1122-1128 (2015).

14. Mirjalili, S., Mirjalili, S.M., and Hatamlou, A. "Multiverse optimizer: a nature-inspired algorithm for global optimization", Neural Computing and Applications, 27(2), pp. 495-513 (2016).

15. Faris, H., Aljarah, I., and Mirjalili, S. "Training feedforward neural networks using multi-verse optimizer for binary classification problems", Applied Intelligence, 45(2), pp. 322-332 (2016).

16. Hu, C., Li, Z., Zhou, T., Zhu, A., and Xu, C. "A multi-verse optimizer with levy flights for numerical optimization and its application in test scheduling for network-on-chip", PLOS ONE, 11(12), e0167341 (2016).

17. Sayed, G.I., Darwish, A., and Hassanien, A.E. "Quantum multi-verse optimization algorithm for optimization problems", Neural Computing \& Applications, 31, pp. $2763-2780$ (2017).

18. Sulaiman, M., Ahmad, S., Iqbal, J., et al. "Optimal operation of the hybrid electricity generation system using multiverse optimization algorithm", Computational Intelligence and Neuroscience, 2019, Article ID 6192980, 12 pages (2019).

19. Karthikeyan, K. and Dhal, P.K. "Multi verse optimization (MVO) technique based voltage stability analysis through continuation power flow in IEEE 57 bus", Energy Procedia, 117, pp. 583-591 (2017).

20. Ahmed, F. and Hegazy, R. "Multi-verse optimizer for identifying the optimal parameters of PEMFC model", Energy, Elsevier, 143(C), pp. 634-644 (2018).

21. Jia, H., Peng, X., Song, W., et al. "Multiverse optimization algorithm based on lévy flight improvement for multithreshold color image segmentation", IEEE Access, 7, pp. 32805-32844 (2019).
22. Hans, R., and Kaur, H. "Binary Multi-Verse Optimization (BMVO) approaches for feature selection", International Journal of Interactive Multimedia and Artificial Intelligence, 6(1), pp. 91-106 (2020).

23. Kennedy, J. and Eberhart, R. "Particle swarm optimization", Proceedings of IEEE International Conference on Neural Networks, Piscataway, NJ, pp. 1942 1948 (1995).

24. Bansal, J.C., Singh, P.K., Saraswat, M., et al. "Inertia weight strategies in particle swarm optimization", Third World Congress on Nature and Biologically Inspired Computing, Salamanca, 2011, pp. 633-640 (2011).

25. Atashpaz-Gargari, E. and Lucas, C. "Imperialist competitive algorithm: An algorithm for optimization inspired by imperialistic competition", 2007 IEEE Congress on Evolutionary Computation, Singapore, pp. 4661-4667 (2007).

26. Abdollahi, M., Isazadeh, A., and Abdollahi, D. "Imperialist competitive algorithm for solving systems of nonlinear equations", Computers \& Mathematics with Applications, 65(12), pp. 1894-1908 (2013).

27. Hosseini, S. and Al Khaled, A. "A survey on the imperialist competitive algorithm metaheuristic: Implementation in engineering domain and directions for future research", Applied Soft Computing, 24, pp. 1078-1094 (2014).

28. Sherinov, Z., Ünveren, A., and Acan, A. "Imperialist competitive algorithm with updated assimilation for the solution of real valued optimization problems", International Journal on Artificial Intelligence Tools, 27(02), p. 1850005 (2018).

29. Mokhtarian Asl, M. and Sattarvand, J. "An imperialist competitive algorithm for solving the production scheduling problem in open pit mine", IJMGE Int. J. Min. \& Geo-Eng., 50(1), pp. 131-143 (2016).

30. Mirjalili, S., Mirjalili, S.M., and Lewis, A. "Grey wolf optimizer", Advances in Engineering Software, 69, pp. 46-61 (2014).

31. Faris, H., Aljarah, I., Al-Betar, M.A., et al. "Grey wolf optimizer: a review of recent variants and applications", Neural Computing and Applications, 30(2), pp. 413-435 (2018).

32. Gao, Z.M. and Zhao, J. "An improved grey wolf optimization algorithm with variable weights", Computational Intelligence and Neuroscience, 2019, Article ID 2981282, 13 pages (2019). https://doi.org/10.1155/2019/2981282 (2019).

33. Yan, F., Xu, J., and Yun, K. "Dynamically dimensioned search grey wolf optimizer based on positional interaction information", Complexity, 2019, Article ID 7189653, 36 pages (2019). https://doi.org/ $10.1155 / 2019 / 7189653$

\section{Biographies}

D. Jamunaa was born in Tamil Nadu, India. She obtained her BE in Electronics \& Communication 
Engineering from Bharathiar University, Coimbatore, MS in Microwave and Optical Engineering from AC College of Engineering and Technology, Karaikudi, and $\mathrm{PhD}$ in the field of Antenna Arrays from NIT, Durgapur, India. She has been involved in teaching subjects related to Electronics and Communication Engineering. Her research areas include antenna arrays and evolutionary algorithms.

G.K. Mahanti was born in India in 1967. He obtained his BE in Electronics \& Communication Engineering from NIT, Durgapur, India, ME in Electronics System and Communication from NIT, Rourkela, India, and PhD (Engg.) from IIT, Kharagpur, India. He has more than 29 years of teaching and research experience. Presently, he is working as a Professor (HAG) at the Department of Electronics and Communication Engineering, National Institute of Technology, Durgapur, India. $\mathrm{He}$ is a senior member of IEEE, USA, and Fellow of Institution of Engineers (India). He has published approximately
110 papers in journals as well as national and international conferences. He was a reviewer in many international journals like Electronics Letter, IEEE Antennas and Wireless Propagation Letter, Progress in Electromagnetics Research, IET Microwave, Antenna and Propagation, and many conferences. He was also the program committee member of many national and international conferences. His research areas include array antenna synthesis, evolutionary algorithms, machine learning, and electromagnetics.

Feras N. Hasoon was born in Basrah, Iraq in 1973. He obtained his $\mathrm{PhD}$ degree in Electrical, Electronics, and System Engineering from University Kebangsaan Malaysia in 2008. He is currently an Assistant Professor at the Department of Electrical and Communication Engineering in College of Engineering, NUST, Sultanate of Oman. He has more than 15 years of academic experience in teaching and research. His research areas include communication systems, optical communications, and coding techniques. 